Review Article

\title{
Human Gut Microbiome Research in India: A Retrospect and Future Opportunities
}

\author{
DHIRAJ P DHOTRE, SHREYAS V KUMBHARE, VILAS P SINKAR and YOGESH S SHOUCHE* \\ National Centre for Microbial Resource, National Centre for Cell Science, Savitribai Phule University of \\ Pune Campus, Ganeshkhind, Pune 411 007, India
}

(Received on 03 April 2018; Accepted on 28 October 2019)

\begin{abstract}
In recent years, human gut microbiome research has moved from being an area of basic research to the advance therapeutics. After the realization of the potential role of the microbiome research in human health, several government agencies and major pharmaceutical companies across the globe have initiated many mega projects. Human microbiome research in India is still in its infancy and a lot needs to be done to understand and investigate the role of microbiota in shaping the overall health and disease conditions especially in the Indian scenario. Indian population harbours tremendous genetic and cultural diversity consistingof more than 6,000 communities and approximately 40,000 endogamous groups. Indian population isstructured and thus endogamous group and geographical origin need to be essentially considered while making any molecular inferences. These variations make Indian population a perfect model to study the 'Genotype-Microbiome' association. This articleretrospectively reviewsthe progress in Indian gut microbiome research, ranging from the population based microbial profiling to the bacterial isolations and their genomic characterization and attempts to put forth the future prospects in this area.
\end{abstract}

Keywords: Human Microbiota; Human Gut Microbiome; Indian Microbiome; Metagenomics; Microbial Diversity

\section{Introduction}

The human body harbors diverse microbial communities, which are predominantly bacterial and referred to as 'Human Microbiome'. According to the current understanding, the human body is inhabited by up to 10 times more microbial cells $\left(\sim 10^{14}\right)$ than human cells $\left(\sim 10^{13}\right)$ (Luckey, 1972; Sleator, 2010; Suau et al., 1999). Microbes contribute 360 times more genes than humans' own genes, which play a key role in human physiology and development. These microbes residing inand on our bodyplay crucial role in many functions, ranging frommetabolism of otherwise complex indigestible carbohydrates and fats to producing essential vitamins along withtrainingour immune system toacting as thefirst line of defense against pathogens (Cho and Blaser, 2012; Turnbaugh et al., 2007).

Extensive research work carried out on the human microbiome in last decade has given insights into several extrinsic and intrinsic factors which contribute in shaping the composition of the microbiome, including genetics, dietary habits, age, geographic location and ethnicity (Cho and Blaser, 2012; Marathe et al., 2012). More importantly, these studies led a strong foundation to decipher its implications on human health (Lloyd-Price et al., 2016) and diseases which range from neonatal health, gastrointestinal disorders, rheumatoid arthritis, diseases associated with skin, lung, liver, urogenital tract, neurological disorders, cancer to lifestyle diseases like obesity and diabetes (Huttenhower et al., 2012; Turnbaugh et al., 2007). Amongst all the extrinsic factors affecting gut microbiome, a long-term diet seems to have the largest effect which is clearly observed from the research studies focusing on the effects of dietary interventions on the gut microbiome (Bhute et al., 2016; Conlon and Bird, 2015; Flint, 2012; Kau et al., 2012; Xu and Knight, 2015). These studies also revealed few important aspects like how different

*Author for Correspondence: E-mail: yogesh@nccs.res.in 
parts of the human body like skin, oral cavity, lungs, gastrointestinal and urogenital tract are occupied by characteristic microbial communities, which vary in the composition between individuals, as well as over the course of a human lifetime. Review of the work done so far indicates a complex tripartite association between genotype, environmental factors like food habit and microbiome.

Indian population harbours tremendous genetic and cultural diversity (Kumar and Reddy, 2003; Misra, 2001). It consists of over 6,000 communities (people of India: http://www.ansi.gov.in) and approximately 40,000 endogamous groups (Majumder, 1998). Basu et al., in 2016 showed that in addition to the Ancestral North Indians (ANI) and Ancestral South Indians (ASI), there are two ancestral components in mainland India that are AAA (for "Ancestral Austro-Asiatic") and ATB (for "Ancestral Tibeto-Burman"). Andaman Islanders retain a fifth distinct ancestry component (Basu et al., 2016). Further pairwise FST (fixation index), a measure of population differentiation due to genetic structure, in Indian population is much larger than that of Europeans, indicating that Indian populations are structured (Reich et al., 2009). Indian population structure is largely contributed by endogamous groups from distinct geographical originand thus these factors need to be considered while making any molecular inferences. An admixture of these endogamousgroups makes Indian population a perfect model to study 'Genotype-Microbiome' association, where genetically linked populations with different dietco-existwith genetically distinct populations with similar diet (Shetty et al., 2013a). Moreover, India harboursa large number of autochthonous tribal populations that are largely unaffected by the 'modern' diet and lifestyle (Bates and Carter, 1992; Shetty et al., 2013a).

While there had been considerable efforts made globally in different aspects of human microbiome research, such associations remain largely unexplored in India. This review makes an attempt to summarize the work carried out in human gut microbiome research in India (Table 1). We have made an attempt to categorize the human gut microbiome research carried out in India in four major arms. Figure 1 illustrates the four major arms of human gut microbiome research carried out in India.

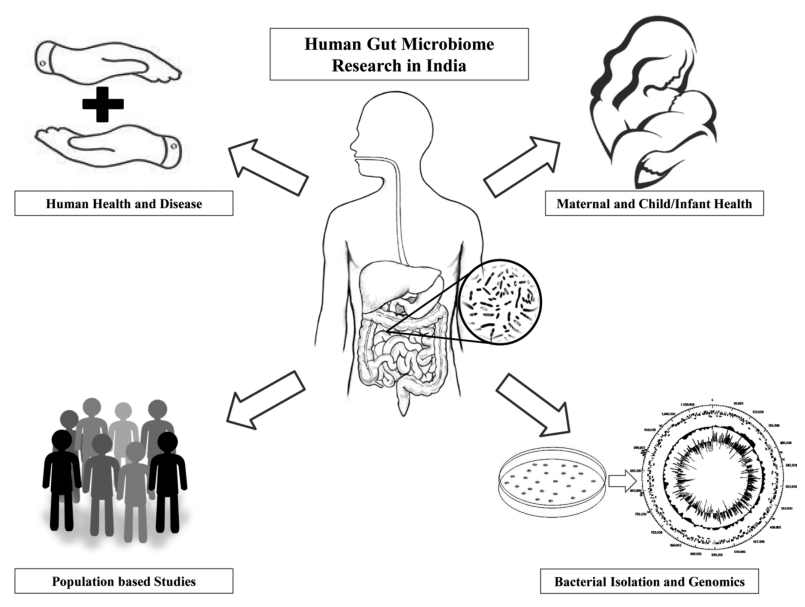

Fig. 1 :A framework for opportunities available in four major areas in human gut microbiome research in India

\section{Population-based Studies: Baseline Data}

In the modern concept of human health, deciphering the composition and functional characteristics of the indigenous microbiome is crucial. Alterations in gut microbiome are well-documented in the disease state, but health associated gut microbiome is largely unexplored. Previous studies on the human gut microbiome primarily focused on identifying the core set of microbial taxa present in healthy individuals, with the assumption that they would lack microbes present in disease phenotypes (Turnbaugh et al., 2007). However, recent high-throughput studies on healthy human microbiome revealed sufficient variation in microbial composition and structure which rapidly disproved the former assumption (Lloyd-Price et al., 2016). Moreover, considering the extent of population-based variation observed, microbiome of healthy individuals cannot be easily extrapolated to more diverse populations around the world. Here we discuss few studies carried out on healthy human microbiome within Indian population.

Marathe et al., 2012, investigated the gut microbiota of two Indian families, each with three individuals belonging to successive generations of the same family. Denaturation gradient gel electrophoresis (DGGE), qPCRand 16S rRNA gene-based clone library analysis was used to understand the microbial communities.Further, isolations and biochemical assays were carried out focusing mainly on strict anaerobes. The study revealed age dependent variation 
Table 1: List of few important studies carried out in the field of human gut microbiome research from India

\begin{tabular}{|c|c|c|c|c|c|}
\hline S.No. & Reference & $\begin{array}{l}\text { Sample size } \\
\text { (only Indian } \\
\text { subjects) }\end{array}$ & Sequence accession no. & Specimen type & Techniques \\
\hline 1 & $\begin{array}{l}\text { (Marathe et al., } \\
\text { 2012) }\end{array}$ & 6 & $\begin{array}{l}\text { JQ264784-JQ265743 } \\
\text { (clone library analysis) }\end{array}$ & Faecal samples & $\begin{array}{l}\text { Isolation of strict anaerobes, clone } \\
\text { library based } 16 \mathrm{~S} \text { rRNA gene sequencing, } \\
\text { DGGE and qPCR }\end{array}$ \\
\hline 2 & (Bhute et al., 2016) & 34 & $\begin{array}{l}\text { Ion Torrent PGM runs } \\
\text { were deposited to NCBI } \\
\text { SRA under the accession } \\
\text { numbers SRP041693, } \\
\text { SRP055407 and Illumina } \\
\text { raw reads to DDBJ under } \\
\text { the accession number } \\
\text { DRA002238 }\end{array}$ & Faecal samples & $\begin{array}{l}\text { 16S rRNA gene sequencing using Ion torrent } \\
\text { PGM and Illumina HiSeq } 2000 \text { sequencing } \\
\text { technologyand qPCR based quantification } \\
\text { of dominant OTUs }\end{array}$ \\
\hline 3 & (Yadav et al., 2016) & 22 & $\begin{array}{l}\text { Downloaded from } \\
\text { public databases }\end{array}$ & Faecal samples & $\begin{array}{l}\text { Meta analyses using } 399 \text { metagenomes from } \\
\text { various studies focusing on microbial network } \\
\text { interactions }\end{array}$ \\
\hline 4 & $\begin{array}{l}\text { (Bhattacharya } \\
\text { et al., 2015) }\end{array}$ & 22 & $\begin{array}{l}\text { Downloaded from } \\
\text { public databases }\end{array}$ & Faecal samples & $\begin{array}{l}\text { Meta analyses using } 448 \text { metagenomes from } \\
\text { different geographies were analysed focusing } \\
\text { on CAZyme profiles }\end{array}$ \\
\hline 5 & (Dehingia et al., 2015) & & & & \\
\hline 6 & (Patil et al., 2012) & 20 & GU954553-GU957822 & Faecal samples & $\begin{array}{l}\text { Quantification of genus Bacteroides and } \\
\text { archaea using qPCR, Clone library based 16S } \\
\text { rRNA gene sequencing }\end{array}$ \\
\hline 7 & $\begin{array}{l}\text { (Suryavanshi et al., } \\
\text { 2016) }\end{array}$ & 39 & $\begin{array}{l}\text { SRP066940 (SRA } \\
\text { submission) }\end{array}$ & $\begin{array}{l}\text { Urine, surgically } \\
\text { removed kidney } \\
\text { stones and fecal } \\
\text { samples }\end{array}$ & $\begin{array}{l}\text { Chemical analysis of kidney stones was } \\
\text { performed using FTIR analysis, oxalate } \\
\text { quantification from } 24 \text { h urine sample } \\
\text { using HPLC, } 16 \mathrm{~S} \text { rRNA gene sequencing } \\
\text { using Ion Torrent PGM, Targeted functional } \\
\text { gene analysis using DGGE, targeted amplicon } \\
\text { (frc-gene) sequencing using Ion torrent PGm } \\
\text { and qPCR }\end{array}$ \\
\hline 8 & (Walujkar et al., 2014) & 6 & DDBJ: DRA001221 & Biopsy samples & $\begin{array}{l}\text { qPCR based quantification of specific species } \\
\text { and } 16 \text { S rRNA gene sequencing using Illumina } \\
\text { HiSeq } 2000\end{array}$ \\
\hline 9 & $\begin{array}{l}\text { (Pushpanathan } \\
\text { et al., 2016) }\end{array}$ & 30 & Not mentioned & $\begin{array}{l}\text { Faecal and serum } \\
\text { samples }\end{array}$ & $\begin{array}{l}\text { Ion torrent PGM sequencing using } \\
\text { 16S rRNA gene } \mathrm{V} 6 \text { region, MCP-1 and IFN } \gamma \\
\text { using ELISA }\end{array}$ \\
\hline 10 & (Ghosh et al., 2014) & 20 & $\begin{array}{l}\text { SRR1067674, SRR } \\
\text { 1087919, SRR1068216, } \\
\text { SRR1067721, SRR106821 } \\
\text { SRR1068218, SRR106821 } \\
\text { SRR1068596, SRR106859 } \\
\text { SRR1067720, SRR106771 } \\
\text { SRR1087910, SRR108791 } \\
\text { SRR1067716, SRR108791 } \\
\text { SRR1087914, SRR108791 } \\
\text { SRR1087916, SRR106771 } \\
\text { SRR1087918 }\end{array}$ & $\begin{array}{l}\text { Faecal samples } \\
17 \\
19, \\
97 \\
19 \\
11 \\
13 \\
15 \\
17\end{array}$ & $\begin{array}{l}\text { Whole shotgun metagenome sequencing } \\
\text { using Pyrosequencing technique }\end{array}$ \\
\hline 11 & (Gupta et al., 2011) & 2 & $\begin{array}{l}\text { Submitted to MG-RAST } \\
\text { server, MG-RAST/ } \\
\text { sequence accession }\end{array}$ & Faecal samples & $\begin{array}{l}\text { Whole metagenome sequencing using } 454 \\
\text { GSFLX pyrosequencing platform (healthy } \\
\text { child sample was sequenced using the }\end{array}$ \\
\hline
\end{tabular}


number not mentioned

12

(Dinh et al., 2016)

13 (Kabeerdoss et al., 2011) 56

14

(Balamurugan et al., 2010a)

15

(Kao et al., 2016)

16

(Pandey et al., 2012a)

17 (Pandey et al., 2012b)

18

(Balamurugan

et al., 2010b)

19

(Kabeerdoss

et al., 2013)

20

(Balamurugan

et al., 2008)
20

10

24

SRP066115 (SRA

accession number)

NA

NA

Venous blood

samples were

taken for the

estimation of $\mathrm{Hb}$

and $\mathrm{Fe}$ and faecal

samples for bacte-

rial quantification

NCBI Short Read Archive Urine, Blood and under Bioproject number samples PRJNA290203

EU723273-EU723399

for VB infants library

Faecal samples

and from EU723400-

EU723483 for CB

infants

4 infants and

4 mothers

14

NA

EU326530 to EU326687 Faecal samples

Faecal samples

83

NA

Faecal samples

130 children NA

and 30 adults
GSFLX chemistry, the malnourished child sample was sequenced using the Titanium chemistry)

16S rRNA gene targeted amplicon sequencing using Illumina MiSeq platform

qPCR based quantification of Clostridium coccoides group (Clostridium cluster XIVa), Roseburia spp.-Eubacteriumrectale, Bacteroides-Prevotella group,

Bifidobacterium genus, Lactobacillus group, Clostridium leptum group (Clostridium cluster IV), Faecalibacteriumprausnitzii, Ruminococcusproductus-C. coccoides, Butyrivibrio, Enterococcus species and Enterobacteriaceae

qPCR based quantification of Eubacteriumrectale, Clostridium leptum group, Bifidobacterium genus, BacteroidesPrevotella-Porphyromonas group and Lactobacillus acidophilus group

LC-MS/MS based measurements of citrulline, ornithine, and arginine, GC-MS based urinary Mannitol concentration measurement, Urinary lactulose measurement using YSI 2300 Stat Plus Analyzer, 16S r RNA gene targeted amplicon sequencing using 454 GSFLX platform

Clone library based 16S rRNA gene sequencing

Clone library based 18S rRNA gene sequencing for micro-eukaryotic diversity

Temporal Temperature Gradient Electrophoresis (TTGE) of 16S rDNA amplified using total bacteria and bifidobacteria specific primers. Real time PCR, targeting 16S rDNA, was used to quantitate faecal bifidobacteria and enterobacteria

qPCR based quantification of Bifidobacterium, Lactobacillus acidophilusgroup, Bacteroides-Prevotella group,

Enterobacteriaceae, Enterococcus, Clostridium coccoides-Eubacteriumrectale group, Clostridium leptum group and Staphylococcus

qPCR based determination of the relative predominance of Bifidobacterium genus, Bacteroides-Prevotella-Porphyromonas group, Lactobacillus acidophilus group, Eubacteriumrectale, and Faecalibacterium prausnitzii 
in gut microbiota amongst the individuals within each family. Based on the higher percentage of novel bacterial speciesand differences in the pattern of Firmicutes /Bacteroidetes ratio with age, this study for the first time suggested that the gut microbiota of Indian individuals is different as compared to the western population.

Extending the earlier investigation, another study by Bhute et al., in 2016 described gut microbial communities of healthy Indian subjects and compared them with the microbiota from other populations. In the meta-analyses carried out in this paper, authors showed that the Indian gut microbiome is different than Americans and is similar to the Bangladeshi population. They also highlighted that the predominance of genus Prevotella and Megasphaera are one of the most distinctive features of Indian gut microbiome. Further, considering the diverse dietary habits of the Indian population, they compared the gut microbiome of Indian samples with non-human primates (with herbivorous, carnivorous and omnivorous diet), revealing high similarity of Indians with omnivorous primates.

In order to understand the distinctive features of healthy Indian gut microbiome, Yadav et al. 2016 performed a meta-analysis on 399 gut metagenomes from 8 different countries and identified certain geography and age-specific trends with respect to composition, intra-group heterogeneity, and diversity of the gut microbiomes. The inter-microbial network analysis carried out in the study showed several crossgeography trends. Along with the specificity of certain bacterial groups only in the older population, an increasing trend of microbial interactions with age was observed. Amongst the 8 inter-microbial networks analysed, the Indian population was observed to be characterized by a distinct decrease in the cooccurrence of genera. The mutual exclusion network for the Indian population was, on the other hand, observed to be sparse with only six genera having four interactions between them.

Bhattacharya et al. 2015 conducted a comprehensive in-silico analysis of Carbohydrates Active enzyme (CAZyme) profiles in the gut microbiota of 448 individuals belonging to different geographies. This study detected several geography and age-specific trends in the gut CAZyme repertoire of the individuals which was also observed to be associated with BMI. Further analysis showed that these digestive CAZymes were majorly contributed by phylum Firmicutes. They found that the abundance of CAZymes is lower in Indian infants/ childrenas compared tothose belonging to Japanese, Venezuela and Malawi population.

Due to the unique diet and almost untouched by the modern lifestyle, studies on gut microbiome of Indian tribal population can provide inimitable links for the evolution of gut microbiome. Dehingia et al. 2015 studied the gut bacterial diversity of the tribes of India to understand the effect of ethnicity and geography on gut microbiota and carried out a metaanalysis to compare them with the worldwide data. Fecal bacterial diversity was studied in fifteen Mongoloid and Proto-Australoid tribal populations representing four geographic regions (Assam, Telangana, Manipur and Sikkim) of India. They showed that geography and diet had a significant effect on gut microbiota of the Indian tribes which was observed to be dominated by Prevotella. Thismetaanalysis revealed that gut microbiota of the Indian population is similar to that of Mongolians.

Indian traditional family structure and endogamy provides a unique opportunity to study these associations in greater details. Although few studies have been carried out to generate baseline data in the Indian context, robustlongitudinal (especially spatial) studies with larger sample size and advanced molecular techniques are required to get decisive outcomes. Previous studies indicate the importance of the gut microbial community of Indian tribes that exhibit functional flexibility and reduced rate of metabolic activities which are connected with many metabolic diseases (Rampelli et al., 2015; Schnorr et al., 2014; Turroni et al., 2016). The prevalence of lifestyle related disorders like obesity, diabetes, IBD etc. has been known to be significantly lower compared to the non-tribal (urbanized) populations across the globe (Jain et al., 2015). This leads the foundation for a larger study on gut metagenome configuration of Indian population including the tribes of India which would be of immense help to improve our knowledge about the evolution of gut microbiota. 


\section{Human Microbiome in Health and Diseases}

Although it is evident that microbes play a key rolein human health, the exact underlying mechanism in the development of disease needs to be fully elucidated. With the current knowledge, it is becoming increasingly apparent that there is sufficient variation in human microbiome across different populations comprising of complex microbial and host interactions. Hence it is difficult to generalize the results of microbiome and disease association studies carried out on a particular population to design any future therapeutics.

Patil et al. 2012 for the first time carried out a comparative analysis and quantification of the dominant gut microbiota of lean, normal, obese and surgically treated obese individuals of Indian origin. A representative gut microbial diversity was assessed by sequencing fecal $16 \mathrm{~S}$ rRNA clone libraries for each group $(\mathrm{n}=5)$ with a total of over 3000 sequences. Bacteroides genus wasfound to be prominent among the obese individuals. In addition, remarkably high archaeal diversity with elevated fecal SCFA levels was also noted in the obese group. The surgically treatedobese individuals exhibited comparatively reduced Bacteroides and archaeal counts along with reduced fecal SCFAs. Surgically treated-obese individuals displayed few notable and relative trends as seen in normal individuals. Comparative decrease in Bacteroides population, along with decrease darchaea and fecal SCFAs are some compelling observations which are somewhat different than the western world.

Walujkar et al. 2014 studied the gut microbiota in patients suffering from different stages of Ulcerative colitis (UC) using 16S rRNA genebased sequencing and qPCR. Authors found a significant reduction in total microbial diversity in UC patients, with an increase in abundance of unusual aerobes and facultative anaerobes, including a member of the phylum Proteobacteria. Also, a 10-fold increase in the total bacterial count was detected in patients suffering from a severe inflammatory stage when compared with patients with moderate and mild stages of inflammation. The reduction of bacterial diversity with an increase in the total bacterial count indicated a shift in bacterial communities which implied dysbiosis and dys-anaerobiosis in severe stages of UC.

The first in-depth surveillance of OMBS in the human gut and their association with hyperoxaluria was investigated by Suryavanshi et al. 2016. Based on 16S rRNA gene amplicons, targeted metagenomic sequencing of Formyl-CoA transferase ( $f r c$ ) gene and qPCR assay, they demonstrated a selective enrichment of Oxalate Metabolizing Bacterial Species (OMBS) in hyperoxaluria condition (Suryavanshi et al., 2016). This study reported thatsignificantly higher concentration of oxalate was inhibitory to many gut microbes, including oxalate metabolizing bacterium, Oxalobacter formigenes. In addition, acid tolerant bacteria were higher in recurrent kidney stone sufferers. Further, specific enzymes participating in oxalate metabolism were significantly abundant in stone sufferers. The study revealedthe association of hyperoxaluria driven dysbiosis with oxalate content, stone episodes and colonization pattern of Oxalobacter formigenes.

Pushpanathan et al.2016 studied the association of gut microbiota with type 2 diabetes mellitus (T2DM) on 30 subjects (17 T2DM and 13 nondiabetics) by using $16 \mathrm{~S}$ rRNA gene-based sequencing. Gram-negative bacteria; Escherichia and Prevotella were found to be dominant in T2DM subjects as compared to the non-diabetic group. Based on Human MCP 1 and IFN $\gamma$ Enzyme-Linked Immunosorbent Assay (ELISA) and 16S rRNA gene sequencing data, they have suggested that lipopolysaccharides present in the cell wall of Gram-negative bacteria might be responsible for low-grade inflammation in the T2DM patients.

These studies focusing on various diseases such as obesity, diabetes, ulcerative colitis and kidney stone demonstrated a significant alteration in gut microbial composition and function. Although these investigations were grounded on few subjects, they intensely indicate the need to carry out further extensive studies to understand the mechanisms and succession of dysbiosis to form a basis for novel therapeutics. Also, these findings suggest a need for future efforts using temporal studies on larger population size which can help in identifying microbial biomarkers.

\section{Microbiome in Maternal and Child Health}

Maternal and child malnutrition/undernutrition is a global problem and is highly prevalent in Sub-Saharan Africa and South Asia, as compared to other regions 
of the world (Black et al., 2013). According to the UNICEF data on monitoring the situation of children and women across the globe in 2015, it was noted that two out of every four stunted children live in South Asia and one in three in Sub-Saharan Africa. The Rapid Survey on Children (RSoC) 2013-2014 based on a nationwide data (inclusive of both urban and rural area) suggest that, inage group 0-59 months, 38.7\% and $17.3 \%$ children in India are observed with stunted (height for age below-2SD) and severely stunted (height for age below-3SD) nutritional status respectively(Ministry of Women and Child Development Government of India, 2014).

From the emerging studies testing the association of gut microbiome and dietary patterns and/or the nutritional status strongly suggest that these gut inhabitants contribute significantly in nutrient assimilation and in turn to the child development. One such study carried out on 20 Indian rural children with varying nutritional status, on the basis of metagenomic data, demonstrated that the microbial communities and their associated functions vary with nutritional status (Ghosh et al., 2014). Moreover, potentially pathogenic groups such as Escherichia, Streptococcus, Shigella etc. were observed to be highly enriched in the severely malnourished children. The essential carbohydrate active enzymes (CAZymes) crucial in energy harvest from complex carbohydrates were seen higher in the healthy children. Additionally, another study carried out by Gupta et al. 2011 also indicated differences in the microbial communities and functions in the gut, when compared between a malnourished and a healthy child. Theyalso showed that the gut of malnourished children harboured bacterial community belonging to order Campylo bacterales with functions like antibiotic resistance and iron metabolism in higher abundance, along with genes responsible for pathogenicity such as Type VI secretion systems.

Longitudinal studies are of prime importance to understand the long-term effects of the factors and monitor the microbial succession in the gut with increasing age. A longitudinal study was carried out by Dinh et al. 2016 to study the alteration in gut microbiota in persistently stunted young children (from birth up to 2 years) in south India. This study demonstrated a higher abundance of phylum Bacteroidetes in stunted children as compared to the control group. The microbiota of control children was enriched in probiotic species Bifidobacterium longum and Lactobacillus mucosae, while few taxa with an inflameogenic property such as Desulfovibrio genus and order Campylobacterales were seen higher in stunted children. In addition, significant alterations in abundance of Bifidobacterium longum and Bifidobacterium pseudolongum (decrease) and Clostridiaceae (increase) were observed with increasing age. Although these studies are based on a very small cohort, the outcomes of the study form a basis for metagenomic studies on malnourished children in India and suggest a need to carry out an extensive study with a larger cohort. However, considering the severity of malnourishment in India, there are very few studies focusing on the gut microbiome aspect which may serve as a potential approach to improve the nutritional status of children in the country.

Extensive research has been carried out on adolescent and maternal health across the globe including India. Previous studies suggest a strong association of maternal nutritional status such as BMI with the fetal or postnatal development. The RSoC 2013-2014 report suggests that around 44.7\% girls aged 15-18 years are of low weight (BMI <18.5). Furthermore, according to the nutrition report of the National Family Health Survey-3 (NFHS-3), almost half (48\%) of Indian women (age 15-49 years) suffer from either abnormal thinness or overweight/ obesity(Arnold and Kothari, 2009). However, considering these facts and evidences of the role of the gut microbiome in health and nutrition, very few studies have focused on deciphering the gut microbial composition and function of women in India. A study carried out by Kabeerdoss et al. 2011 compared and showed a difference in gut bacterial composition between vegetarian and non-vegetarian (omnivorous diet) women. This study based on the real-time quantification showed that Clostridium cluster XIVa (specifically Roseburia-E. rectale) were enriched in women with an omnivorous diet, which also exhibited high correlation with the microbial butyryl-CoA CoAtransferase gene. Additionally, few research studies have also made an attempt to understand the gut functions such as microbiome mediated arginine metabolism and iron absorption. A study carried out by Balamurugan et al. 2010 describes the association 
of gut bacterial community and iron-deficiency anaemia in women aged 18-25 years. The research study demonstrated a significantly lower abundance of Lactobacilli in anaemic women as compared to the normohaemic group, indicating an association of gut bacterial community and iron absorption. Further, another study focused on a tripartite association of gut microbiome, intestinal function and arginine metabolism in Indian women as compared to American and Jamaican women (Kao et al., 2016). This study compared the gut microbiome and intestinal function of non-pregnant women of childbearing age; findings suggest that Indian women have decreased citrulline flux and higher enteral hydrolysis of arginine as compared to other groups. It was noted that intestinal absorptive capacity and endogenous arginine flux was higher in Bacteroides- dominant subject group (American and Jamaican) as compared to Prevotelladominant Indian subject group, moreover these differences correlated with the dietary, gut function and metabolic parameters of each population group. However, considering that these studies are based on real time quantification of few bacterial groups and very low sample size, larger studies with highthroughput molecular techniques are required to draw subtle inferences about such associations within Indian population.

Exposure to microbes and their colonization in the human intestine begins right from the fetal development and is enhanced again during birth by maternal contact. It is also evident from previous studies that early exposures such as breastfeeding and vaginal contact during birth play an important role in modulating and shaping the microbiome in infants (Black et al., 2013). This microbial colonization with beneficial bacteria in the early life helps in the development of immune system. Moreover, other extrinsic factors such as nutrition, hygiene, etc. serve as key drivers for alterations in microbiome (Black et al., 2013). Thus, assessment of maternal gut microbial diversity pre and post pregnancy is considered to be of utmost importance. Also, understanding the association of various factors shaping the early infant gut flora is equally important, which may serve as the basis for diagnostic or further intervention approaches to improve the overall health of children.

Based on the IYCF (Infant and Young Child Feeding) practices details from the RSoC and the
NFHS-3 reports, of the total children aged 0-23 months only $44.6 \%$ were breastfed immediately/ within an hour of birth, while almost one-third of infants less than two months of age are not being exclusively breastfed in India (Ministry of Women and Child Development Government of India, 2014). Additionally, around $49 \%$ of children of age 6-59 months are moderately anaemic as per the NFHS-3 report (Arnold and Kothari, 2009). Considering these facts and the importance of early assessment of microbiome in infancy, diagnostic and interventional studies to modulate and improve the gut microbial colonization of beneficial microbes in mothers and infants may improve the overall health. Also, such approaches would prove to be helpful in the prevention of several gastrointestinal diseases like infantile colic and Necrotizing Enterocolitis (NEC). Few important studies carried out to understand the maternal and child gut microbiome during pregnancy and early infants within Indian population, provide basic leads about gut functions and microbial community and their association with various factors.

One of the early studies carried out by Pandey et al., 2012 to investigate differences in the gut bacterial diversity of infants born through vaginal and caesarean delivery. This study for the first time in Indian infants (24 infants) using the cultureindependent technique, showed differences in the gut bacterial composition of caesarean and vaginally born infants. Specifically, it reported higher Acinetobacter sp., Bifidobacterium sp. and Staphylococcus sp. in vaginally born infants, while Citrobacter sp., while Escherichia coli and Clostridium difficilewere dominant in caesarean born infants. Another study carried out by Pandey et al. 2012 to detect the presence of micro-eukaryotic diversity in formula fed and breastfed infants and their respective mothers revealed that the maternal gut flora consisted diverse micro-eukaryotes such as Blastocystis sp., Saccharomyces sp., Candida albicans etc. as compared to their infants which harboured no detectable micro-eukaryotes. Further, Balamurugan et al. 2010 made an attempt to decipher the effect of asymptomatic rotavirus infection on the gut Bifidobacterial and Enterobacterial abundance in the early stage of infancy (first month). This study based on the Temporal Temperature Gradient Electrophoresis (TGGE) of the 16S rDNA amplicons, 
demonstrated that the infection was not associated with alterations in the abundance of Bifidobacteria and Enterobacteria in the first month of life. However, this study represents an important effort in understanding the alterations in the early gut microbial flora of infants, which eventually is a key driving force for immuno-modulation and development with long term-effects on human health.

Moreover, few other studies carried out by Kabeerdoss et al. 2013 and Balamurugan et al. 2008 investigated the development of gut microbial composition in the first six months and succession during childhood and adolescence exhibiting the importance of conducting longitudinal assessments in children. The research study focusing on the gut bacterial alterations of 83 infants in first six months revealed that bacterial members of Entero bacteriaceae, Bifidobacteria, Lactobacilli, and Staphylococci are predominant in early stages of life (till 28 days), while in the later stage (3-6 months) dominance is taken over by Bacteroides-Prevotella and $C$. coccoides- $E$. rectale. Additionally, it also provided insights into the association of factors such as birth mode and feeding pattern with the gut microbiome in early stages of life. These findings were investigated for their long term effects during adolescence and adulthood by Balamurugan et al. 2008. This assessment of bacterial succession in 130 healthy Indian children suggested that there are significant changes in the abundance of bacteria such as Lactobacillus, Bifidobacterium, Bacteroides, E. rectale and $F$. prausnitzii. The former two being dominant in the early stage undergo a gradual decrease with increasing age, while the rest increase and are found highest during the late childhood in the Indian population (Balamurugan et al., 2008). It reported that gradual changes take place in the gut bacterial flora after the early childhood and these changes are also observed till the adolescence and adulthood. These studies although based on targeting specific bacterial groups in the gut using the qPCR technique, emphasize the need of extensive research work with larger and well-monitored cohorts in India. Nonetheless, these attempts have generated a basis to design further investigations using advanced techniques like high-throughput sequencing platforms. Channelized and standardized pipelines for the assessment of the gut microbial diversity of mothers and children in India, with an aim of generating new diagnostic and therapeutic approaches, are required for the improvement of maternal and child health. Undertaking a large study may lead to the generation of maternal and child microbial profiles from diverse ethnic, socioeconomic groups with a wide range of dietary habits and patterns. This baseline information can help researchers in designing further interventional studies for mother and children in India.

\section{Bacterial Isolation and Genomics}

Recent advancements in culturomic studies have clearly suggested the importance of isolation and characterization of human gut microbes in designing future therapeutic approaches. Genome sequencing using high-throughput platforms isproviding deeper insights into the functional potential of bacterial strains (Greub, 2012). Very less proportion of the gut bacterial diversity is represented by cultured isolates due to the challenges involved in culturing them in the laboratory. Recently, integrated approach of genomics, metagenomics, and metabolomics along with culturomics has proved to be immensely useful in growing previously uncultured gut bacteria (Dickson, 2016; Lagier et al., 2012). These multi-omic techniques have not only led to the discovery of novel taxa but have also enabled researchers to explore the functional potential of these gut inhabitants (Dickson, 2016). Unfortunately, very few studies on novel taxa and genome sequencing of gut bacterial isolates have been reported from the Indian subcontinent.

To the best of our knowledge, only four novel gut bacterial taxa have been validly reported and published from India, as described in Table 2. These include Clostridium Punense (Lanjekar et al., 2016a), Megasphaera indica (Lanjekar et al., 2016b), Pantoea intestinalis (Prakash et al., 2015) and Pelistegaindica (Prakash et al., 2014). The former two being strict anaerobes while the later are reported to be aerobic isolates. These strains were isolated from human faeces of healthy individuals. Further, as of yet, the genome sequence of 10 gut bacterial strains, constituting 7 bacterial taxa have been reported and validly published from India (see Table 3 ). These studies indicate that the gut bacterial isolate harbour a wide range of gene repertoire for nichespecific functions such as bile and acid tolerance, Carbohydrate-Active enzymes (CAZymes), 
Table 2: List of novel taxa isolated from human gut in India

\begin{tabular}{|c|c|c|c|c|c|}
\hline S.No. & Novel Taxa & $\begin{array}{l}\text { Accession number } \\
\text { (16S rRNA nucleo- } \\
\text { tide sequence) }\end{array}$ & $\begin{array}{l}\text { Culture deposition } \\
\text { details }\end{array}$ & Description & Reference \\
\hline 1 & $\begin{array}{l}\text { Clostridium } \\
\text { punense }\end{array}$ & JN093128 & $\begin{array}{l}\text { DSM } 28650 \\
\text { CCUG } 64195 \\
\text { MCC } 2737\end{array}$ & $\begin{array}{l}\text { Strict anaerobe, Gram-stain positive, spore } \\
\text { forming bacterium isolated from healthy } \\
\text { human fecal sample }\end{array}$ & $\begin{array}{l}\text { (Lanjekar et al., } \\
\text { 2015) }\end{array}$ \\
\hline 2 & $\begin{array}{l}\text { Megasphaera- } \\
\text { indica }\end{array}$ & $\begin{array}{l}\text { Strain NMBHI-10 } \\
\text { (HM990965) } \\
\text { Strain BLPYG-7 } \\
\text { (HM990964) }\end{array}$ & $\begin{array}{l}\text { StrainBLPYG-7 } \\
\text { (DSM 25563, } \\
\text { MCC 2481) } \\
\text { Strain NMBHI-10 } \\
\text { (DSM 25562, } \\
\text { MCC 2482) }\end{array}$ & $\begin{array}{l}\text { Strict anaerobe, Gram-stain-negative, } \\
\text { coccoid, both strains isolated from } \\
\text { fecal samples of two healthy humans }\end{array}$ & $\begin{array}{l}\text { (Lanjekar et al., } \\
\text { 2016) }\end{array}$ \\
\hline 3 & $\begin{array}{l}\text { Pantoeaintes- } \\
\text { tinalis }\end{array}$ & KP326384 & $\begin{array}{l}\text { DSM } 28113 \\
\text { MCC } 2554\end{array}$ & $\begin{array}{l}\text { Aerobic, Gram-stain negative, non-spore } \\
\text { former, rod-shaped bacterium isolated from } \\
\text { human fecal sample of healthy individual }\end{array}$ & $\begin{array}{l}\text { (Prakash et al., } \\
\text { 2015) }\end{array}$ \\
\hline 4 & Pelistegaindica & KF692037 & $\begin{array}{l}\text { MCC } 2185 \\
\text { DSM } 27484\end{array}$ & $\begin{array}{l}\text { Aerobic and non-fastidious, Gram-stain } \\
\text { negative, coccoid, Nitrate reducing bacteria } \\
\text { isolated from human fecal sample of healthy } \\
\text { individual }\end{array}$ & $\begin{array}{l}\text { (Prakash et al., } \\
\text { 2014) }\end{array}$ \\
\hline
\end{tabular}

production of Short Chain Fatty Acids (SCFA) and important metabolites such as vitamins and essential amino acids (Shetty et al., 2013b). Extensive characterization of the indigenous probiotic strain has gained attention in recent years. Lactobacillus plantarum strain Lp91 (Grover et al., 2013a) and Lactobacillus fermentum strain Lf1 (Grover et al., 2013b) are two such indigenous strains isolated and sequenced from Indian human gut. These studies reveal the presence of gene clusters involved in diverse probiotic functions indicating their potential to be used as efficient indigenous probiotics for Indian population.

Genome sequencing has been used as an effective tool to characterize various human pathogens. This approach has improved our knowledge of the intricacies of pathogenicity of several bacterial isolates. Few studies reported from India on genome sequencing and annotation of gut bacterial isolates helped us understand their pathogenic characters such as virulence patterns, multi-drug resistance etc. A study carried out by Marathe et al. 2014 on genomic characterization of Clostridium sp. isolated from human gut, showed the presence of various genes responsible for Multi-Drug Resistance (MDR). These genes specifically included MDR-type $\mathrm{ABC}$ transporters, drug/metabolite transporters
(DMT), multidrug and toxin extrusion (MATE) family efflux pumps. Additionally, the research studies carried out by Kulkarni et al. 2013 and Kulkarni et al. 2014 on Ochrobactrum intermedium strains revealed the pathogenic potential of these bacteria in the host. The genomic data obtained from these studies demonstrated that they possess various gene clusters such as urease, secretion systems and flagellar genes sharing high similarity with Helicobacter pylori, a known pathogen of the gastrointestinal tract in humans. Genomic studies have also been carried out on Shigella dysenteriae (Kaur et al., 2014) and Vibrio fluvialis (Khatri et al., 2013) strains to determine the pathogenic characters of these gut pathogens responsible for gastric diseases such as shigellosis and diarrhoea respectively. The genome analysis of Vibrio fluvialis showed that apart from the virulence genes, a gene cluster (EutBCEGPR) for Ethanolamine (EA) utilization was present, which was also confirmed using growth analysis experiment.Further, in the other study on Shigella sp., metabolic pathway analysis showed that $S$. dysenteriae strain SD1D possessed high tendency to be pathogenic due to the acquisition of antibiotic resistance genes from external sources. In addition, the strain also carried a wide range of genes responsible for multi-drug resistance and heavy metal resistance, indicating its potential to survive in the 
stress conditions like an antibiotic or heavy metal stress (Khatri et al., 2013). The comparative genomics approach used in these studies demonstrates the differences and similarities in closely related pathogenic strains. These findings suggest that these gut pathogens have flexible genomes providing a higher chance of adaptability to the environment, enabling them to survive in stress conditions such asthe presence of heavy metals, antibiotic stress, acidic conditions etc.

In summary, efforts of isolating human gut bacteria will complement targeted amplicon studies by providing the information about their functional capabilities. The newly emerging approach of culturomics, which essentially involves extensive isolation and characterization of microbes from various ecological niches, can serve as an efficient technique to study the human gut microbiome with a deeper dimension (Lagier et al., 2012). Further, the isolations and genome sequencing provide an opportunity to mine the metabolic profile of these bacteria and theirassociation with host physiology and metabolism. As discussed earlier, the human gut microbiota alters with confounding factors such as diet and genetic make-up. Culturomicsapproach will surely be useful in exploring novel taxa with novel functional capabilities from the Indian tribes, which are very rarely exposed to the modern lifestyle.

\section{Current Understanding and Future Endeavors}

The voluminous work carried across the globe in the last decade indicates that there are substantial differences in microbiome in different parts of the world (Bhute et al., 2016; Fettweis et al., 2014; Nam et al., 2011; Nishijima et al., 2016). Studies on Japanese and Chinese population postulated the possible existence of hitherto unknown factors contributing to the population-level diversity in human gut microbiome (Chong et al., 2015; Leung et al., 2015; Nishijima et al., 2016). From the outcomes of the work done so far, it is evident that Indian population harbours a diverse and distinct microbiota, much due to the differences in dietary habits and lifestyle as compared to the western world. The diverse Indian population serves as an interesting potential model to explore the human microbial diversity.

Considering the uniqueness of Indian gut microbiome, it is imperative to establish a link between disease status and alteration of gut microbiota using much larger cohorts. A collection of extensive metadata of the study subjects is necessary to understand the effect of intrinsic and extrinsic factors in altering the gut microbiota and progression of the disease. Health and demographic surveillance systems (HDSS) available in India can be exploited to carry out longitudinal studies on larger homogenous population (Ghosh et al., 2015). HDSS setup can overcome the limitations experienced in microbiome research, such as statistically significant sampling size, long-term follow-up, and collection of comprehensive metadata.

Maternal and child health assessment and improvement are one of the most important factors for healthy development and overall betterment of the society. There is a need for comprehensive studies with a vision of understanding the role of gut microbiota in iron and folic acid deficiency, maternal and child undernutrition along with the effect of factors such as suboptimal/no breastfeeding, birth mode and early exposure to antibiotics. Microbiome research can aid in the development of various nutrition-specific interventions and programs to improve maternal and child health.

To the best of our knowledge, very few bacterial isolates and genome sequences are reported so far from Indian gut samples (Table 2 and 3). Hence there is a need for establishment and standardization of culturomics studies to isolate and characterize gut microbes from Indian population. Identification and cataloguing of these isolates will help in developing microbial consortia for gut microbial modulation and therapy of a particular disease, as a substitute to nebulous methods such as whole fecal transplants. Thus, there is a clear scientific opportunity to carry out a comprehensive study to explore the microbial diversity within Indian population, and test its association with various factors like diet, genotype, lifestyle, hygiene etc. Such data can be used extensively to explain the pattern that would emerge by exploring confounding factors which may be responsible for the observed variation. Figure 2 explains the broad framework for opportunities available in human gut microbiome research in India. A retrospect of the human gut microbiome research in India clearly suggests its huge scientific potential in future efforts to decipher the microbiome-based 
Table 3: List of human gut bacterial genomes published from India along with their important genomic features

\begin{tabular}{|c|c|c|c|}
\hline S. No. Gut isolate & $\begin{array}{l}\text { Genome Accession } \\
\text { numberDDBJ/ } \\
\text { EMBL/GenBank }\end{array}$ & Description & Reference \\
\hline
\end{tabular}

$1 \quad$ Clostridium punense AUPA00000000 strain BL8 from human gut. Genome also encodes for type IV secretion system with presence of PilABC and PilMNO proteins and pilus regulator PilR

Multidrug resistant novel clostridium species isolated (Marathe et al., 2014)
2 Lactobacillus $\quad$ AXDQ00000000 plantarum strain Lp91
$3 \quad$ Lactobacillus fermen- tum strain Lf1

\section{AWXS00000000}

Megasphaeraindica Strain NM10: strain NM10 and strain BL7 APHY00000000 Strain BL7: APHX00000000

$5 \quad$ Ochrobactrum Strain 229E: intermedium strain ASXJ00000000 229E and strain M86

Strain M86: AOGE00000000

$6 \quad$ Shigelladysenteriae

AURX00000000 strain SD1D

$7 \quad$ Vibrio fluvialis strain PG41 and strain I21563
Isolate with probiotic potential isolated from human gut. (Grover et al., 2013a) Isolate exhibits various probiotic potentials such as High acid, bile tolerance etc.

Isolate with probiotic potential isolated from human gut. (Grover et al., 2013b) Isolate possess probiotic attributes. Inhibitory activity against gut pathogens

Human gut isolates with bile resistance genes, stress response systems and with diverse range of

(Shetty et al., 2013) Carbohydrate Active enzymes (CAZymes)

Emerging opportunistic pathogen, concurrent with (Kulkarni et al., 2013; Helicobacter pylori. Harbors gene clusters for virulence, Kulkarni et al., 2014) stress response and urease production, Isolated from human gut

Shigellosis (acute form of gastroenteritis) causing

(Kaur et al., 2014) pathogen. Harbored 37 genes encoding functional

Clinical strains isolated from human gut, Diarrheal

(Khatri et al., 2013) pathogen. Harbors ethanolamine utilization protein encoding genes proteins for pathogenicity
Strain PG41: ASXS01000000 Strain I21563: ASXT01000000

\section{Human Gut Microbiome}

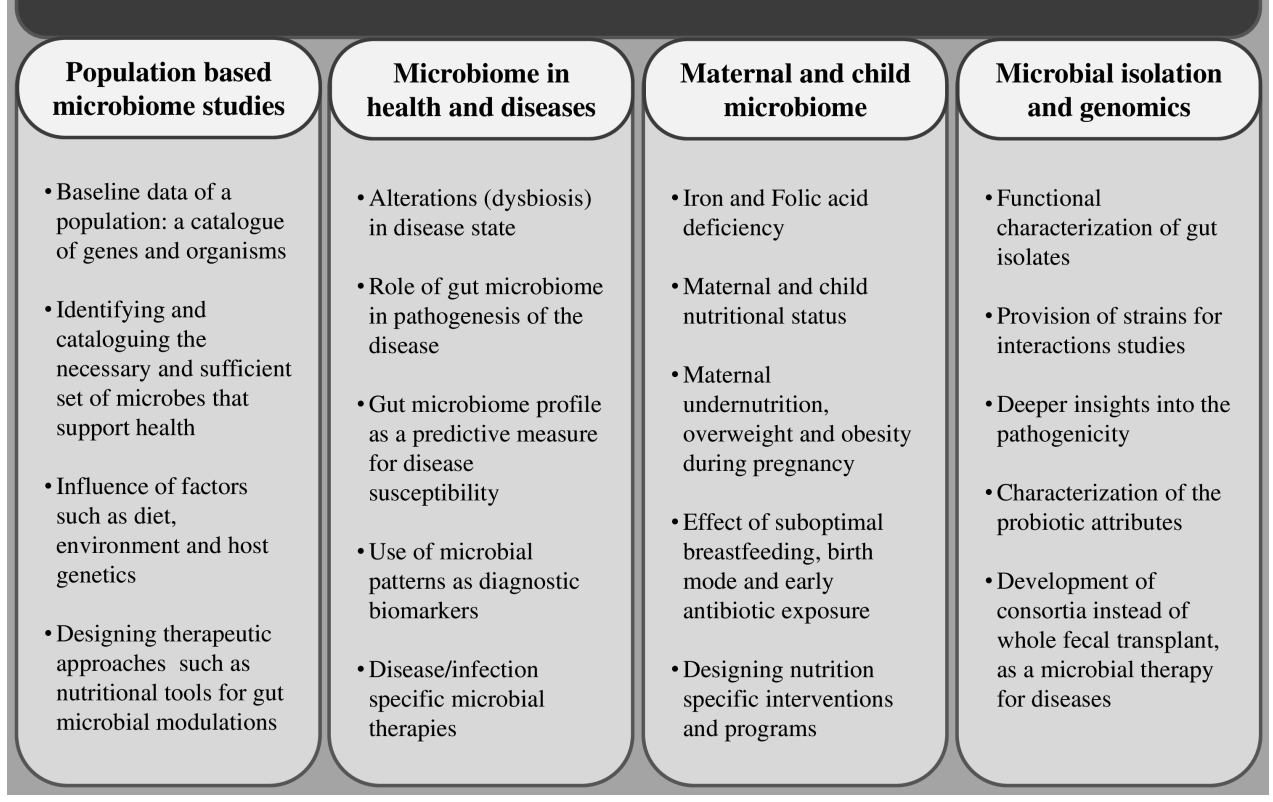

Fig. 2 : A Broad framework for opportunities available in human gut microbiome research in India 
solutions to improve the health of the Indian population. These few, but important studies have led us to understand how the compositionally distinct and diverse microbiome responds to various confounding factors such as diet, age and disease state. Also, they have further facilitated the discovery of previously unknown principles including the influence of various factors such as disease, maternal diet, and health on the gut microbial ecosystem of individuals in the underexplored Indian population. As also mentioned earlier, considering the urge of developing microbiome and/or diet-based tools for modulating the health status worldwide, these research studies clearly indicate that the outcomes of the western research cannot be implied or generalized on the Indian population. Hence, one needs to carefully consider these recently learned facts while utilizing the well-explored western interventional models for Indian population. Moving further, despite these developments, there is a need for a comprehensive vision to undertake an interdisciplinary research to understand the underlying mechanisms of microbes in the key process such as human body homeostasis. Recently emerging characterization studies using the functional genomics approach also hold a great potential. However, the lack of culturomics based microbiome studies is the bottleneck to develop promising indigenous and efficient probiotics. Establishment of a biobank

\section{References}

Arnold F and Kothari SPPAM (2009) Nutrition in India

Balamurugan R, Janardhan HP, George S and Chittaranjan SP (2008) Bacterial succession in the colon during childhood and adolescence: molecular studies in a southern Indian village 1 - 3 Am J Clin Nutr 88 1643-1647

Balamurugan R, Mary RR, Chittaranjan S, Jancy H, Devi RS and Ramakrishna BS (2010a) Short Communication Low levels of faecal lactobacilli in women with iron-deficiency anaemia in south India Br J Nutr 104 931-934

Balamurugan R, Magne F, Balakrishnan D, Suau A, Ramani S, Kang G and Ramakrishna BS (2010b) Faecal bifidobacteria in Indian neonates \& the effect of asymptomatic rotavirus infection during the first month of life Indian J Med Res $132721-727$

Basu A, Sarkar-Roy N and Majumder PP (2016) Genomic reconstruction of the history of extant populations of India reveals five distinct ancestral components and a complex comprising of such potential isolates is necessary for broad range investigations ranging from the screening of beneficial bioactive compounds to their utilization in newly emerging 'bacteriotherapy' in the dysbiosis state.

\section{Conclusions}

The growing evidence of the distinctness of human microbiome of Indian subjects both compositionally and functionally as compared to the western world, suggests that there await highly unexplored and untapped fundamental aspects in Indian microbiome science. Microbiome research in India has undoubtedly unmasked an important field in the human health sector and now needs to be taken further with a robust setup of the state-of-the-art facilities and high-end computational methodologies. Notably, there exist several decisive challenges and thus indicates an urge of extensive understanding of the human health in India from microbiome perspective.

In summary, studies with robust sampling design accounting for genetic, cultural and biogeographic diversity are required which will lead to the genesis of fundamental and comprehensive understanding of the Indian Human Microbiome, eventually benefiting the medical and health care system.

structure Proc Natl Acad Sci USA 113 1594-1599

Bates C and Carter M (1992) Tribal Migration in India and Beyond World Rural Labourer Colon India 310

Bhattacharya T, Ghosh TS and Mande SS (2015) Global profiling of carbohydrate active enzymes in human gut microbiome PLoS One 10 1-20

Bhute S, Pande P, Shetty SA, Shelar R, Mane S, Kumbhare S, Gawali A, Makhani H, Navandar M, Dhotre D, et al. (2016) Molecular characterization and meta-analysis of gut microbial communities illustrate enrichment of Prevotella and Megasphaera in Indian Subjects Front Microbiol 7 1-14

Black RE, Victora CG, Walker SP, Bhutta ZA, Christian P, Onis M De and Ezzati M (2013) Maternal and Child Nutrition 1 Maternal and child undernutrition and overweight in low-income and middle-income countries Lancet 382 427451

Cho I and Blaser MJ (2012) The human microbiome: At the 
interface of health and disease Nat Rev Genet 13 260-270

Chong CW, Ahmad AF, Lim YAL, Teh CSJ, Yap IKS, Lee SC, Chin YT, Loke P and Chua KH (2015) Effect of ethnicity and socioeconomic variation to the gut microbiota composition among pre-adolescent in Malaysia Sci Rep $\mathbf{5}$ $13338-13350$

Conlon MA and Bird AR (2015) The Impact of Diet and Lifestyle on Gut Microbiota and Human Health 17-44

Dehingia M, Thangjam Devi K, Talukdar NC, Talukdar R, Reddy N, Mande SS, Deka M, and Khan MR (2015) Gut bacterial diversity of the tribes of India and comparison with the worldwide data Sci Rep 518563

Dickson I (2016) Culturomics: Illuminating microbial dark matter Nat Rev Gastroenterol Hepatol 14189

Dinh DM, Ramadass B, Kattula D and Sarkar R (2016) Longitudinal Analysis of the Intestinal Microbiota in Persistently Stunted Young Children in South India Longitudinal Analysis of the Intestinal Microbiota in Persistently Stunted Young Children in South India Plos One 5 e 0155405-17

Fettweis JM, Paul Brooks J, Serrano MG, Sheth NU, Girerd PH, Edwards DJ, Strauss JF, Jefferson KK and Buck GA (2014) Differences in vaginal microbiome in African American women versus women of European ancestry Microbiol (United Kingdom) 160 2272-2282

Flint HJ (2012) The impact of nutrition on the human microbiome Nutr Rev7010-13

Ghosh TS, Gupta SS, Bhattacharya T, Yadav D, Barik A, Chowdhury A, Das B, Mande SS and Nair GB (2014) Gut microbiomes of Indian children of varying nutritional status PLoS One 9 1-13

Ghosh S, Barik A, Majumder S, Gorain A, Mukherjee S, Mazumdar S, Chatterjee K, Bhaumik SK, Bandyopadhyay SK, Satpathi B, et al. (2015) Health \& Demographic Surveillance System Profile: The Birbhum population project (Birbhum HDSS) Int J Epidemiol 44 98-107

Greub G (2012) Culturomics: A new approach to study the human microbiome Clin Microbiol Infect 18 1157-1159

Grover S, Sharma VK, Mallapa RH and Batish K (2013a) Draft Genome Sequence of Lactobacillus plantarum Strain Lp91, a Promising Indian Probiotic Isolate of Human Gut Origin Genome Announc 1 e00976-13

Grover S, Sharma VK, Mallapa RH and Batish K (2013b) Draft Genome Sequence of Lactobacillus fermentum Lf1, an Indian Isolate of Human Gut Origin Genome Announc 1 e00883-13

Gupta SS, Mohammed MH, Ghosh TS, Kanungo S, Nair GB and
Mande SS (2011) Metagenome of the gut of a malnourished child Gut Pathog 3 1-9

Huttenhower C, Fah Sathirapongsasuti J, Segata N, Gevers D, Earl AM, Fitzgerald MG, Young SK, Zeng Q, Alm EJ, Alvarado L, et al. (2012) Structure, function and diversity of the healthy human microbiome Nature $\mathbf{4 8 6}$ 207-214

Jain Y, Kataria R, Patil S, Kadam S, Kataria A, Jain R, Kurbude R and Shinde S (2015) Burden \& pattern of illnesses among the tribal communities in central India: A report from a community health programme Indian JMed Res 141663 72

Kabeerdoss J, Devi RS, Mary RR and Ramakrishna BS (2011) Short Communication Faecal microbiota composition in vegetarians: Comparison with omnivores in a cohort of young women in southern India Br J Nutr 108 953-957

Kabeerdoss J, Ferdous S, Balamurugan R, Mechenro J, Vidya R, Santhanam S, Jana AK and Ramakrishna BS (2013) Development of the gut microbiota in southern Indian infants from birth to 6 months: a molecular analysis $J$ Nutr Sci 2 1-7

Kao CCC, Cope JLL, Hsu JWW, Dwarkanath P, Karnes JMM, Luna RAA, Hollister EBB, Thame MMM, Kurpad AVV and Jahoor F (2016) The microbiome, intestinal function, and arginine metabolism of healthy indian women are different from those of American and Jamaican women $J$ Nutr 146 1-8

Kau AL, Ahern PP, Griffin NW, Goodman AL and Jeffrey I (2012) Human nutrition, thegut microbiome, and immune system: envisioning the future Nature 474 327-336

Kaur G, Sathyabama S, Arora A, Verma S, Mubin N and Agrewala JN (2014) Genome sequencing, annotation and comparative genomic analysis of Shigella dysenteriae strain SD1D Gut Pathog 6 1-6

Khatri I, Mahajan S, Dureja C, Subramanian S and Raychaudhuri S (2013) Evidence of a new metabolic capacity in an emerging diarrheal pathogen: Lessons from the draft genomes of Vibrio fluvialis strains PG41 and I21563 Gut Pathog 5 1-7

Kulkarni G, Dhotre D, Dharne M, Shetty S, Chowdhury S, Misra V, Misra S, Patole M and Shouche Y (2013) Draft genome of Ochrobactrum intermedium strain M86 isolated from non-ulcer dyspeptic individual from India Gut Pathog 5 15

Kulkarni GJ, Shetty S, Dharne MS and Shouche YS (2014) Genome sequencing analysis reveals virulence-related gene content of Ochrobactrum intermedium strain 229E, a urease-positive strain isolated from the human gastric niche 359 12-15 
Kumar V and Reddy BM (2003) Status of Austro-Asiatic groups in the peopling of India: An exploratory study based on the available prehistoric, linguistic and biological evidences J Biosci 28 507-522

Lagier J, Armougom F, Million M, Hugon P, Pagnier I, Robert C, Bittar F, Fournous G and Gimenez G (2012) Microbial culturomics/ : paradigm shift in the human gut microbiome study Clin Microbiol Infect 18 1185-1193

Lanjekar VB, Marathe NP, Shouche YS and Ranade DR (2016a) Clostridium punense sp nov, an obligate anaerobe isolated from healthy human faeces Int J Syst Evol Microbiol $\mathbf{6 5}$ 4749-4756

Lanjekar VB, Marathe NP, Ramana VV, Shouche YS and Ranade DR (2016b) Megasphaera indica sp nov, an obligate anaerobic bacteria isolated from human faeces faeces Int $J$ Syst Evol Microbiol 64 2250-2256

Leung MHY, Wilkins D and Lee PKH (2015) Insights into the pan-microbiome: skin microbial communities of Chinese individuals differ from other racial groups Sci Rep $\mathbf{5} 11845$

Lloyd-Price J, Abu-Ali G and Huttenhower C (2016) The healthy human microbiome Genome Med 851

Luckey TD (1972) Introduction to intestinal microecology Am J Clin Nutr 25 1292-1294

Majumder PP (1998) People of India: Biological diversity and affinitiesof complex human disorders Evol Anthropol Issues, News, Rev 6 100-110

Marathe NP, Shetty SA, Lanjekar V, Shouche YS and Ranade D (2012) Changes in human gut flora with age: An Indian familial study BMC Microbiol 12222

Marathe NP, Shetty SA, Lanjekar VB and Rasane MH (2014) Genome sequencing of multidrug resistant novel Clostridium sp BL8 reveals its potential for pathogenicity $61-5$

Ministry of Women and Child Development Government of India (2014) Rapid Survey on Children

Misra VN (2001) Prehistoric human colonization of India J Biosci 26 491-531

Nam Y Do, Jung MJ, Roh SW, Kim MS and Bae JW (2011) Comparative analysis of korean human gut microbiota by barcoded pyrosequencing PLoS One 6 e22109

Nishijima S, Suda W, Oshima K, Kim SW, Hirose Y, Morita H and Hattori M (2016) The gut microbiome of healthy Japanese and its microbial and functional uniqueness $D N A$ Res 23 125-133

Pandey PK, Verma P, Kumar H, Bavdekar A, Patole MS and Shouche YS (2012a) Comparative analysis of fecal microflora of healthy full-term Indian infants born with different methods of delivery (vaginal vs cesarean): Acinetobacter sp prevalence in vaginally born infants $J$ Biosci 37 989-998

Pandey PK, Siddharth J, Verma P, Bavdekar A, Patole MS and Shouche YS (2012b) Molecular typing of fecal eukaryotic microbiota of human infants and their respective mothers J Biosci 37 221-226

Patil D, Dhotre DP, Chavan S, Sultan A, Jain DS, Lanjekar VB, Gangawani J, Shah P, Stodkar J, Shah S, et al. (2012) Molecular analysis of gut microbiota in obesity among Indian individuals $J$ Biosci 37 647-57

Prakash O, Munot H, Nimonkar Y, Sharma M, Kumbhare S and Shouche YS (2014) Description of Pelistega indica sp nov, isolated from human gut Int J Syst Evol Microbiol 64 1389-1394

Prakash O, Nimonkar Y, Vaishampayan A, Mishra M, Kumbhare S, Josef N and Shouche Y S (2015) Pantoea intestinalis sp nov, isolated from the human gut Int J Syst Evol Microbiol $653352-3358$

Pushpanathan P, Srikanth P, Seshadri KG, Selvarajan S, Pitani RS, Kumar TD and Janarthanan R (2016) Gut Microbiota in Type 2 Diabetes Individuals and Correlation with Monocyte Chemoattractant Protein 1 and Interferon Gamma from Patients Attending a Tertiary Care Centre in Chennai, India Indian J Endocrinol Metab 20 523-530

Rampelli S, Schnorr SLL, Consolandi C, Turroni S, Severgnini M, Peano C, Brigidi P, Crittenden ANN, Henry AGG and Candela M (2015) Metagenome Sequencing of the Hadza Hunter-Gatherer Gut Microbiota Curr Biol 25 1682-1693

Reich D, Thangaraj K, Patterson N, Price AL and Singh L (2009) Reconstructing Indian population history Nature 461 489U50

Schnorr SL, Candela M, Rampelli S, Centanni M, Consolandi C, Basaglia G, Turroni S, Biagi E, Peano C, Severgnini M, et al. (2014) Gut microbiome of the Hadza hunter-gatherers Nat Commun 53654

Shetty SA, Marathe NP and Shouche YS (2013a) Opportunities and challenges for gut microbiome studies in the Indian population Microbiome 124

Shetty SA, Marathe NP, Lanjekar V, Ranade D and Shouche YS (2013b) Comparative genome analysis of Megasphaera sp reveals niche specialization and its potential role in the human gut PLoS One 8 e 79353

Sleator RD (2010) The human superorganism - Of microbes and men Med Hypotheses 74 214-215

Suau A, Bonnet GIS, Sutren ME, Godon J, Gibson GR, Collins MD and Dore J (1999) Direct Analysis of Genes Encoding 
16S rRNA from Complex Communities Reveals Many Novel Molecular Species within the Human Gut Appl Environ Microbiol 65 4799-4807

Suryavanshi MV, Bhute SS, Jadhav SD, Bhatia MS, Gune RP and Shouche YS (2016) Hyperoxaluria leads to dysbiosis and drives selective enrichment of oxalate metabolizing bacterial species in recurrent kidney stone endures Sci Rep 634712

Turnbaugh PJ, Ley RE, Hamady M, Fraser-Liggett CM, Knight $\mathrm{R}$ and Gordon JI (2007) The human microbiome project Nature 449 804-10

Turroni S, Rampelli S, Centanni M, Schnorr SL, Consolandi C, Severgnini M, Peano C, Soverini M, Falconi M, Crittenden
AN, et al. (2016) Enterocyte-Associated Microbiome of the Hadza Hunter-Gatherers Front Microbiol 7865

Walujkar SA, Dhotre DP, Marathe NP, Lawate PS, Bharadwaj RS and Shouche YS (2014) Characterization of bacterial community shift in human Ulcerative Colitis patients revealed by Illumina based 16S rRNA gene amplicon sequencing Gut Pathog 622

$\mathrm{Xu} \mathrm{Z}$ and Knight R (2015) Dietary effects on human gut microbiome diversity Br J Nutr 113 1-5

Yadav D, Ghosh TS and Mande SS (2016) Global investigation of composition and interaction networks in gut microbiomes of individuals belonging to diverse geographies and agegroups Gut Pathog 817. 